
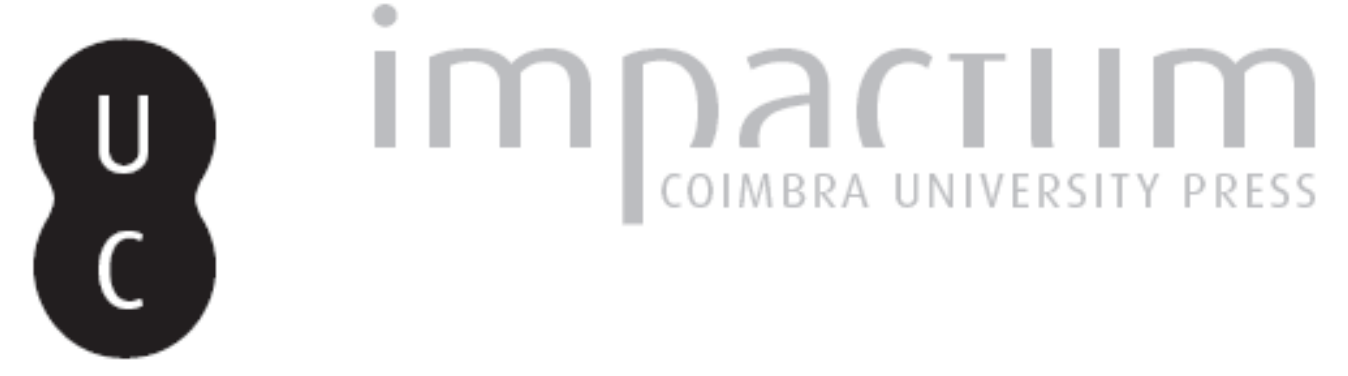

\title{
[Recensão a] João Marinho dos Santos, José Manuel Azevedo e Silva, Mohammed Nadir Santa Cruz do Cabo de Guer d'Agoa de Narba. Estudo e Cronica
}

Autor(es): $\quad$ Coelho, Maria Helena da Cruz

Publicado por: Centro de História da Sociedade e da Cultura

URL

persistente:

URI:http://hdl.handle.net/10316.2/39676

DOI:

DOI:http://dx.doi.org/10.14195/1645-2259_8_9

Accessed : $\quad$ 26-Apr-2023 08:45:30

A navegação consulta e descarregamento dos títulos inseridos nas Bibliotecas Digitais UC Digitalis, UC Pombalina e UC Impactum, pressupõem a aceitação plena e sem reservas dos Termos e Condições de Uso destas Bibliotecas Digitais, disponíveis em https://digitalis.uc.pt/pt-pt/termos.

Conforme exposto nos referidos Termos e Condições de Uso, o descarregamento de títulos de acesso restrito requer uma licença válida de autorização devendo o utilizador aceder ao(s) documento(s) a partir de um endereço de IP da instituição detentora da supramencionada licença.

Ao utilizador é apenas permitido o descarregamento para uso pessoal, pelo que o emprego do(s) título(s) descarregado(s) para outro fim, designadamente comercial, carece de autorização do respetivo autor ou editor da obra.

Na medida em que todas as obras da UC Digitalis se encontram protegidas pelo Código do Direito de Autor e Direitos Conexos e demais legislação aplicável, toda a cópia, parcial ou total, deste documento, nos casos em que é legalmente admitida, deverá conter ou fazer-se acompanhar por este aviso.

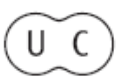




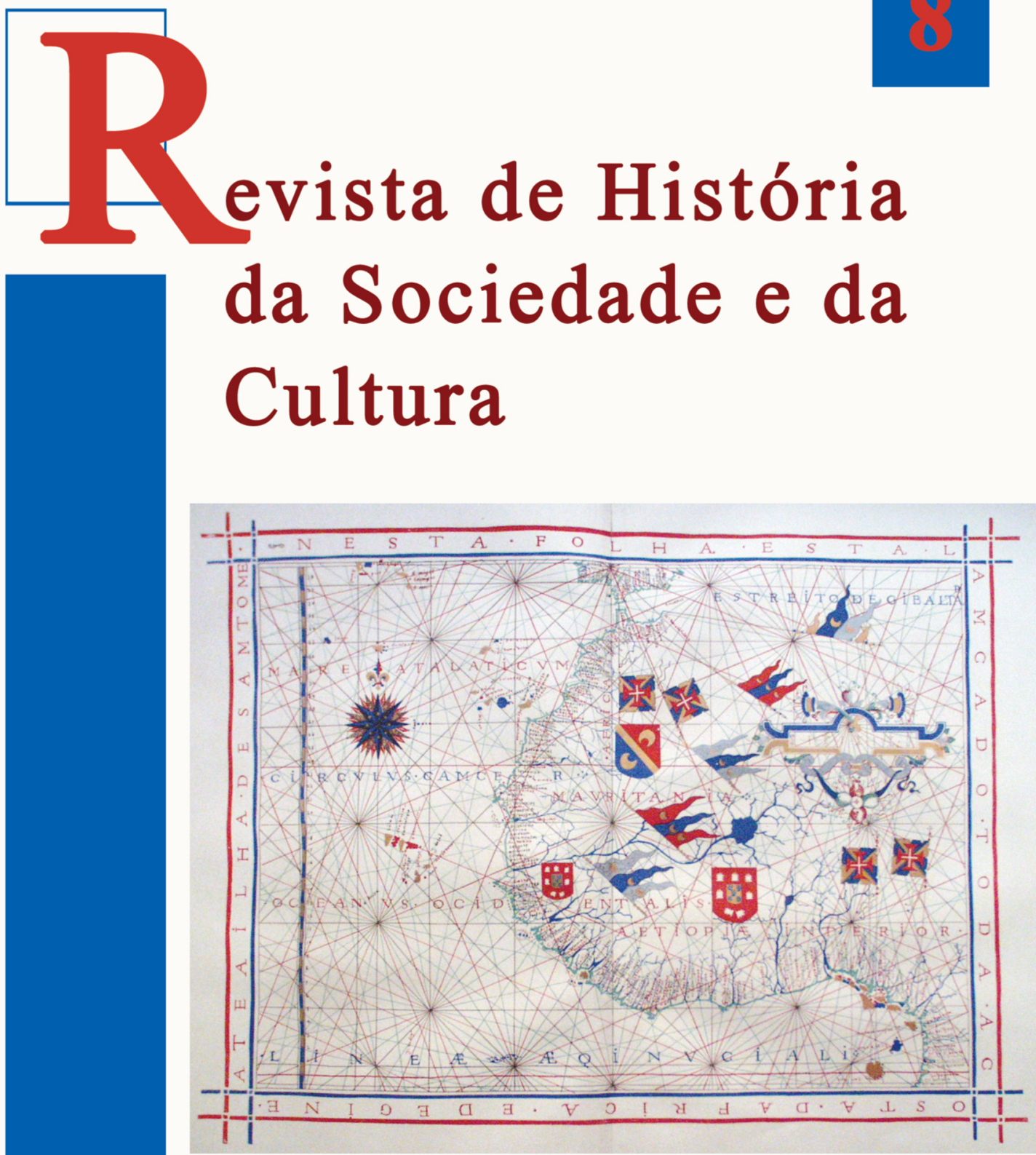

Centro de História da Sociedade e da Cultura Universidade de Coimbra

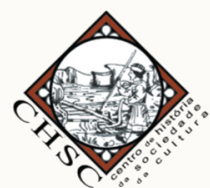




\section{Recensões}

\section{João Marinho dos Santos, José Manuel Azevedo e Silva, Mohammed Nadir Santa Cruz do Cabo de Guer d'Agoa de Narba. Estudo e Cronica, Coimbra, Palimage, 2008.}

J'ai le plaisir de vous présenter un livre consacré à Santa Cruz de Cabo de Guer d'Agoa de Narba, appelée de nos jours Agadir. L'ouvrage, rassemblant des études et une chronique, est rédigé en portugais et en arabe. Cette dualité linguistique manifeste d'emblée une ferme volonté de collaboration entre historiens portugais et marocains.

Le Portugal et le Maroc, partageant un passé largement commun - et qui n'a pas toujours été pacifique - désirent, en ce début du XXle siècle, instituer un nouveau dialogue, ouvert et sans complexes. Seule, il faut le dire, une connaissance approfondie, une confrontation franche de ce passé peut permettre de construire le moment présent, qui est déjà le passé de demain.

L'étude de João Marinho dos Santos, par laquelle s'amorce le livre, «Portugal e Marrocos - da confrontação à cooperação» s'inspire de cette idée même: Portugal et Maroc, de la confrontation à la coopération.

Nous sommes heureusement loin de l'époque où «chrétiens» et «maures» croisaient le fer, dans les champs pierreux de la Péninsule Ibérique ou dans les plaines de sable du Magreb Occidental. Le temps nous a fait passer d'une situation d'hostilités permanentes à une coopération intelligente, plus digne de deux pays voisins qui ont tout à gagner à cultiver la paix et à collaborer dans tous les domaines.

Avant tout atlantiques, par leur position géographique dominante, c'est par la Grande Mer Océane que le Portugal et le Maroc ont établi leurs premiers 
contacts, marqués par une inimitié d'origine essentiellement religieuse. Soumis cependant au milieu méditerranéen, nos deux pays enracinent leurs civilisations et cultures respectives dans ce même fond méditerranéen. Sans parler d'autres points communs comme l'Afrique Noire ou le Proche-Orient, c'est en effet la Méditerranée qui à la fois les sépare et les unit. L'Union Européenne pourra et devra représenter un nouvel espace de dialogue entre le «Nord» et le «Sud», et, singulièrement, entre le Portugal et le Maroc.

Dans des pages d'une grande vigueur démonstrative, João Marinho dos Santos, pour replacer le lecteur dans le contexte spécifique des récits de la Crónica de Santa Cruz de Guer d'Agoa de Narba, essaye d'expliquer la présence des Portugais au Maroc de 1415 à 1541, année où le Portugal commence à perdre, sous l'effet d'une politique globale ou impériale, presque toutes ses places-fortes d'Afrique du Nord. L'auteur rappelle que c'est afin de résoudre des problèmes vitaux (économiques, sociaux et politiques), dans le contexte de la longue dépression européenne et de ses conséquences dans la Péninsule Ibérique, que le roi Jean 1er de Portugal a décidé la conquête de Ceuta. Mais un tel projet visait à constituer un «domaine» (avec d'autres villes, bourgades et territoires proches), qui permettrait au Portugal, à la faveur d'une croissance territoriale et économique, de résister à la menace d'annexion par le royaume de Léon-Castille, dont la puissance s'imposait à toutes les Espagnes. C'est donc pour assurer sa survie nationale que le Portugal a entrepris son expansion, en se lançant dans la conquête de l'Afrique du Nord.

Isolée et constamment menacée de retomber entre les mains des rois de Fez, Ceuta, la principale ville conquise, ne resta portugaise et chrétienne qu'en raison de l'intérêt que la Papauté montrait pour sa possession. C'est en 1458 seulement dans une conjoncture marquée par la perte de Constantinople (1453), qu'Alphonse V de Portugal renoua avec une politique de conquêtes en Afrique du Nord, parallèlement à la forte expansion portugaise dans les Iles de l'Atlantique et en Guinée.

Ainsi après avoir défendu son royaume, le Portugal est devenu le constructeur d'un Empire qui s'est étendu de l'Ancien Monde (Europe, Afrique et Asie) au Nouveau-Monde (les Amériques). Cependant manquant de ressources humaines et financières, incapable de conserver un aussi vaste 
empire, il éprouva bientôt de graves difficultés en Afrique du Nord lorsque, encore sous le règne d'Emmanuel 1er (l'instigateur de la découverte de la route maritime vers l'Inde et du Brésil), les chérifs du Maroc lancèrent, à partir de Sous, leur mouvement de conversion religieuse, d'unification politique et d'expulsion des étrangers.

L'étude de João Marinho dos Santos, d'un côté, nous fait accompagner les Portugais dans leurs efforts pour se maintenir sur un territoire relativement proche et passablement riche et, d'un autre côté, les chérifs marocains dans leur combat pour rassembler, politiquement, leurs frères (en particulier, les «Maures de paix», alliés des Portugais) et pour détrôner le roi de Fez et s'assurer l'appui des Turcs ottomans qui poursuivaient alors leur expansion en Occident.

Santa Cruz do Cabo de Guer fut la première place portugaise à céder, mais c'est là qu'eut lieu ce qui est peut-être la plus grande histoire d'amour entre un Maure et une Chrétienne. Dona Mécia, fille d'un capitaine de Santa Cruz, conquit le coeur du Chérif de Sus, ce qui lui valut de mourir du sortilège lancé par ses rivales, épouses délaissées du Chérif. L'amour peut naître de la guerre! C'est pourquoi le Portugal, dans ses légendes, se souvient de femmes maures ensorcellées, de longues liaisons passionnées, toujours contrariées, clandestines ou durement punies. À l'ère de la globalisation, l'amitié et la coopération entre les peuples sont, certes, moins romanesques mais nous préparent un meilleur avenir!

Dans l'étude suivante, intitulée «O Sus e Santa Cruz do Cabo de Iguir (Agadir)», Mohammed Nadir, en se fondant sur les descriptions laissées par les géographes arabes et des chroniqueurs et voyageurs européens, commence par nous donner une synthèse de la réalité géographique, économique, sociale et politique du Sous, des temps médiévaux jusqu'au XVIe siècle. Son récit est illustré par une suggestive carte physico-économique du Haut-Atlas Occidental, du Sous et de Guzula.

Relevant l'importance stratégique de la place luso-marocaine de Santa Cruz do Cabo de Iguir (autrement dit Agadir Larba), intégrée dans une vaste et riche région du Sous, il en retrace l'histoire depuis sa fondation, en 1505, à sa reddition, en 1541, à travers des aspects divers et significatifs comme:

- l'installation de la forteresse portugaise, en 1505, aux frais d'un particulier, João Lopes de Sequeira, dûment autorisé par le roi; 
- l'intense activité de ce capitaine dans les relations établies dans la région du Sous et les difficultés ressenties pour installer et défendre la place dans les premières années de son existence, jusqu'à son achat par le monarque portugais, Emmanuel, en 1513;

- l'explication du toponyme Santa Cruz do Cabo de Guer d' Agoa de Narba;

- l'action du premier capitaine nommé par le roi, D. Francisco de Castro, et de chacun des capitaines qui lui succédèrent dans la défense et le gouvernement de la place;

- l'abondance de céréales, viande, cuirs, poisson, miel, cire, sucre, coton, indigo, herbes médicinales et autre marchandises provenant du Soudan (or, esclaves, ivoire, piment rouge, poivre);

- l'existence et le fonctionnement du marché, et plus concrètement de la foire hebdomadaire à Santa Cruz do Cabo de Iguir (Agadir);

- l'activité commerciale des Portugais et d'autres Européens (Gênois, Espagnols, Français), ainsi que la présence de contrebandiers, de pirates et de corsaires;

- l'action des chérifs et les raisons de la chute de la place, le 12 mars 1541.

Après cette contextualisation politique et socio-économique, l'Auteur se concentre sur la topographie de la place de Santa Cruz de Cabo de Guer et cherche à reconstituer et à représenter ce patrimoine construit en trois temps distincts.

Ainsi, avant l'arrivée des Portugais, il existait sur le site ou dans ses environs immédiats un espace fortifié ou un magasin de marchandises (en berbère Iguir / Agadir [Agoa]) où se tenait un marché ou une foire tous les mercredis [Narba ou Larbaâ]. Ce qui signifie que la désignation toponymique originelle définissait le type de patrimoine construit ainsi que ses fonctions et activités économiques.

La forteresse construite par João Lopes de Sequeira se composait du château, dans l'enceinte de ses tours, et de deux tourelles (celle de l'Eglise et celle sur la mer). Cette citadelle s'avéra rapidement trop petite et fragile, insuffisante pour loger en sécurité les habitants et les vivres, la garnisation militaire, les commerçants et leurs marchandises.

En 1513, la place passant sous l'autorité directe de la Couronne, le monarque, Emmanuel, fit reconstruire la forteresse pour en faire un ensemble plus vaste et 
plus facile à défendre. Comme la plupart des fortifications portugaises d'outre-mer, elle remplissait une double fonction: à la fois forteresse et comptoir, elle se composait d'un château avec son donjon, d'une enceinte plus étendue constituée de murailles hautes et solides, dominées par sept bastions avec leur artillerie (celui de Taramaque, celui du Château, celui du Fanal, celui de S. Simon, celui des Fours à Chaux, celui du Quartier des Maures, celui del'Eglise). A l'intérieur des remparts se trouvait la cité, avec ses alignements de maisons où logeaient la population et les commerçants, ses boutiques et magasins où étaient conservés les vivres, denrées et autres biens matériels. La place, dans le complexe réseau de ses bâtiments, était complétée par le port et son mouillage, donnant directement accès à la forteresse par la porte de la mer.

L'auteur évoque aussi les destructions subies par la forteresse et les reconstructions rendues nécessaires par les attaques des Maures, notamment lors du terrible siège de 1533 et de l'assaut final de 1541. Il explique encore les intentions du Chérif de Sous, lorsqu'il procéda à la construction du Fort du Pic (Al Kasba Sâadida) en 1540, sur le mont dominant l'agglomération, à 250 mètres d'altitude, appelé aujourd'hui par la population locale Agadir Oufela, ce qui en portugais serait rendu par «Agadir de Cima», Agadir la Haute. Ce fort était composé d'une grande tour et de bastions, où furent installées environ 50 pièces d'artillerie. Trois décennies après, en 1572, la Casbah du Pic sera reconstruite par le Chérif, attestant l'influence de l'architecture militaire portugaise.

L'étude de Mohamed Nadir se termine par une synthèse sur les contacts et l'acculturation entre Marocains et Portugais.

Il faut observer que la véritable raison de ce livre est le document qui s'y trouve reproduit: une chronique de la présence des Portugais dans la place de Santa Cruz de Cabo de Guer (Agadir), depuis sa fondation, en 1505, jusqu'à sa chute, en 1541 .

José Manuel Azevedo e Silva, dans une étude sur «Le manuscrit, l'auteur et le contenu de la chronique», décrit en détail ce spécimen d'archives et nous donne à connaître les problèmes que son pose son attribution ainsi que les lignes essentielles de la chronique.

Nous savons donc que le manuscrit fut identifié pour la première fois en 1775 , lorsque on établit le premier catalogue de la Bibliothèque du Monastère 
d'Alcobaça, qui constitue aujourd'hui un des principaux fonds de la Section des Manuscrits et des Réservés de la Bibliothèque Nationale de Lisbonne. Il fait partie d'un ensemble de 388 folios, portant le numéro 452 du catalogue ancien, devenu le numéro 119 du nouveau catalogue, inséré entre les folios 86 et 136. Il a été publié dans une édition française par Pierre de Cenival en 1934.

Le manuscrit, qui n'est pas signé, ne mentionne pas le nom de son auteur. Mais il est possible de caractériser le personnage qui l'a rédigé, à partir de certains éléments internes, quand il s'exprime à la première personne du singulier, notamment à la fin du texte. On s'aperçoit ainsi que l'auteur était le fils d'un «chevalier fort honoré» qui accompagna le premier capitaine de cette place, Francisco de Castro, en 1513.

On peut se demander si l'auteur vint encore enfant au Maroc avec son père ou s'il y aura plus tard et encore très jeune suivi sa mère. C'est en tout cas dans la place qu'il grandit, s'entraîna à l'exercice des armes, fut probablement armé chevalier. Quand la place tomba, le 12 mars 1541, il était, nous confesse-t-il, au nombre des captifs.

Ce chevalier-chroniqueur tient à citer ses sources d'information. Les faits qu'il a enregistrés, relatifs aux vingt premières années de l'histoire de la place, époque qu'il n'a pas vécue personnellement, ou durant laquelle en tout cas il n'a pas directement participé aux opérations militaires et autres, lui a été racontée par son père et par d'autres hommes «de crédit et de vérité». En ce qui concerne les conflits chez les Maures, notamment les guerres entre les chérifs de Marrakech et de Sous, et autres, dit-il, «menus incidents», il a retenu ce qu'il en a «appris auprès d'honorables cavaliers maures et de prisonniers chrétiens».

Le chroniqueur insiste sur la véracité des faits qu'il rapporte et exerce son esprit critique sur les rumeurs ou les récits qu'il tient de tierces personnes qu'il ne juge pas dignes de foi. En rédigeant sa chronique, entre 1560 et 1570, l'auteur utilise une méthode simple, mais efficace et rigoureuse: il se fait une règle de présenter les aspects généraux, les grands cadres factuels, les illustrant ensuite avec un ou plusieurs épisodes, où ressortent la vivacité et le réalisme des détails.

Relativement au contenu de l'oeuvre, José Manuel Azevedo e Silva nous dit qu'il s'agit, de manière générale, d'une narration des faits que le chroniqueur a estimé devoir figurer dans une histoire de la place de Santa 
Cruz do Cabo de Guer, entre 1505 et 1541. En guise de fil conducteur il entretisse les faits en cherchant à refléter la réalité vécue dans cette place et jusqu'à la région du Sous, durant la période où se succédèrent ses neufs capitaines, de D. Francisco de Castro à D. Guterres de Monroi.

Il n'a garde d'oublier le rôle des femmes dans la vie quotidienne et jusque dans la défense de la place. Les femmes, en plus de soutenir leur famille, appuyaient moralement et matériellement les soldats en temps de guerre. Elles partageaient avec eux les joies de la victoire comme elles se désespéraient de voir partir ou mourir époux et fils. C'est elles encore qui ravitaillaient les hommes au combat, leur apportant aliments, boissons, poudre, armes et munitions. Et, si besoin était, elles s'occupaient des blessés. Elles aidaient encore à transporter des pierres et d'autres matériaux pour contribuer à la reconstruction rapide des murailles endommagées par l'ennemi: le chroniqueur note à ce propos qu'une femme, bien qu'enceinte de sept mois, ne s'est pas dérobée à cette tâche dont dépendait le salut commun. Plus audacieusement encore, les femmes circulaient sur les remparts, munies de casques et de piques (ou de lances), relayant les soldats et montrant à l'ennemi que, derrière ses créneaux, la forteresse ne manquait pas de gens pour la défendre.

Il importe toutefois de remarquer, en conclusion, que les inimitiés, les férocités, les haines entre Chrétiens et Maures n'ont empêché entre eux ni l'amitié, ni la solidarité, ni la loyauté, ni la passion amoureuse. L'auteur anonyme de la chronique publiée dans ce volume a pris soin de rapporter certains de ces épisodes chargés de sentiments et d'émotions.

Les trois textes sont illustrés par des gravures du Maroc, de la région du Sous et spécialement de la bourgade et de la forteresse de Santa Cruz do Cabo de Guer (Agadir), avant sa destruction par le tremblement de terre de 1960. Ce sont ces images qui ont été projetées au cours de cette présentation.

La transcription de la chronique, en portugais et en arabe, s'accompagne de l'indispensable appareil critique où ont été adoptés des critères de transcription favorisant la compréhension du texte sans lui faire perdre sa saveur archaïque ni sa signification culturelle.

Tel est, dans ses grandes lignes, le dessin de l'oeuvre que João Marinho dos Santos, Mohammed Nadir et José Manuel Azevedo e Silva nous ont 
donnée, dans une double version, portugaise et arabe. Et ils ont tenu à venir la présenter eux-mêmes au Maroc.

La chronique ici publiée est un autre témoignage, un autre monumentum du long passé partagé par les Marocains et les Portugais. Un morceau de notre patrimoine commun, riche d'édifices militaires ou religieux, d'objets, d'écrits ou de traditions encore vivantes. C'est un patrimoine précieux qu'il importe de faire connaître, de conserver et d'étudier.

La divulgation de cette chronique et son étude approfondie attestent la volonté de travailler ensemble à la découverte de notre lointain passé, éclairé par nos regards croisés, gage de relations solides et fécondes entre nos pays, aujourd'hui et demain.

\section{Maria Helena da Cruz Coelho}

\section{Présentation de l'ouvrage de José Manuel Azevedo e Silva - Mazagão.} Uma cidade luso-marroquina deportada para a Amazónia, Viseu, Palimage Editores, 2007

Dans l'étude qui sert d'introduction à cet ouvrage, José Manuel Azevedo e Silva, retrace sous une forme synthétique 1'histoire de la place luso-marocaine de Mazagan, de sa fondation par le roi Emmanuel, en 1514, à sa chute, en 1769. Il cherche ensuite à accompagner ses habitants dans leur voyage à Lisbonne, dans leur déportation en Amazonie, dans leur séjour à Belém do Para, jusqu'à leur transfert à la Nouvelle-Mazagan, Nova Mazagão, sur la rive gauche de l'estuaire de l'Amazone. Cette odyssée ne s'est pas achevée là puisque l'actuelle ville de la Nova Mazagão, au Brésil, est située dans un espace différent du Magazan-le-Vieux, Magazão Velho, où avaient été installée la première colonie.

A travers les sources historiques et l'étude qu'il en fait, l'auteur du livre vise, fondamentalement, à accompagner et à expliquer les singuliers avatars de cette population itinérante. Les villes, bourgades et villages détruits, disparus, évacués et déplacés n'ont pas manqué dans l'histoire de l'Humanité, pour toutes sortes de raisons. Mais Mazagan est un cas unique quand on considère les tribulations de ses habitants. 